Trauma Berufskrankh $2006 \cdot 8$ [Suppl 2]:

S212-S217

DOI 10.1007/s10039-005-1061-3

Online publiziert: 1. Dezember 2006

(c) Springer Medizin Verlag 2006

A. Dávid

Zentrum für Unfallchirurgie und Orthopädische Chirurgie, Helios-Klinikum, Universitätsklinikum Witten/Herdecke, Wuppertal

\title{
Operative Therapie der distalen Radiusfraktur
}

\section{Frakturklassifikationen}

figsten Bruch des Erwachsenen dar. Während bis weit in die 1990er Jahre eine konservative Behandlung und die minimalinvasive Bohrdrahtosteosynthese mit anschließender Ruhigstellung über 4-6 Wochen die Behandlungsregimes dominierten, hat sich in den letzten 10 Jahren ein deutlicher Trend zur operativen Therapie mit anschließender funktioneller Nachbehandlung entwickelt [7]. Mit diesen Techniken können kürzere Verweildauern sowie reduzierte Arbeitsunfähigkeitszeiten erzielt werden. Zudem erhöht die funktionelle Nachbehandlung den Komfort für den Patienten.

\section{Diagnostik}

In den meisten Fällen reichen Röntgenaufnahmen des Handgelenks in 2 Ebenen aus, um die Verletzung und das Ausmaß zu beurteilen und eine Klassifikation vorzunehmen.

Bei sehr starker Beteiligung der Gelenkfläche ( $\mathrm{C}_{2}$ - bis $\mathrm{C}_{3}$-Frakturen) kann eine CT-Untersuchung die Analyse erleichtern. Sie kann auch bei begleitenden knöchernen und Bandverletzungen der distalen Handwurzelreihe bedeutsam werden, die bei C-Frakturen in 30-60\% der Fälle beobachtet werden [6].

Die MRT-Untersuchung gehört noch nicht zu den Standardverfahren und kann auch wegen der heute noch eingeschränkten Verfügbarkeit nicht als Routineuntersuchung bei distalen Radiusfrakturen empfohlen werden.
Im deutschsprachigen Raum wird überwiegend die Einteilung der $\mathrm{AO}$ verwendet [7]. Alternativ wird an einigen Zentren die Klassifikation von Frykman bevorzugt.

\section{Indikationen zur Operation}

\section{A-Frakturen}

A1-Frakturen können, sofern sie unverschoben sind, sicher konservativ behandelt werden.

Bei A2-Frakturen kann nach Reposition ebenfalls eine konservative Therapie angestrebt werden. Wesentlich ist, dass es gelingt, die dorsale und palmare Kortikalis sicher aufeinander zu verankern oder achsgerecht minimal einzustauchen [1].

Bei A3-Frakturen muss wegen der zumeist dorsal liegenden Trümmerzone häufig mit einer Redislokation nach Reposition gerechnet werden. Auch bei initial achsgerechten Verhältnissen ist die sekundäre Dislokation dieses Bruchtyps mit Abkippen des distalen Fragments nach dorsal sehr häufig. Daher werden diese Frakturen zunehmend operativ behandelt.

\section{B-Frakturen}

Unverschobene Frakturen des Processus styloideus oder dorsale Kantenabsprengungen (B2-Frakturen) eignen sich für eine konservative Behandlung.

Die palmaren Absprengungen (reversed Barton, $\mathrm{B}_{3}$ ) sollten durch eine Abstützosteosynthese therapiert werden.

\section{C-Frakturen}

Absolut unverschobene stabile intraartikuläre Frakturen können zunächst konservativ behandelt werden. Bleibt die Stellung bei den nachfolgenden Röntgenkontrollen unverändert, kann die konservative Therapie fortgeführt werden.

Liegt eine Stufenbildung vor, die $2 \mathrm{~mm}$ überschreitet, wird grundsätzlich eine Operation empfohlen. $\mathrm{C}_{2}$ - und $\mathrm{C}_{3}$-Frakturen stellen eine absolute Operationsindikation dar.

\section{Verfahren}

Neben der Bohrdrahtosteosynthese können isolierte Schraubenosteosynthesen, volare und dorsale Plattenosteosynthesen sowie die Behandlung mit einem Fixateur externe in Kombination mit einer internen Fixationstechnik durchgeführt werden.

\section{Schraubenosteosynthese}

Verschobene A2-Frakturen, die nach einer Reposition in eine gute stabile Situation gebracht werden können, können perkutan durch eine Schraubenosteosynthese versorgt werden.

Technik. Nach geschlossener Reposition erfolgt über Hautinzisionen eine Zugund Stellschraubenosteosynthese. Die Positionierung der Schrauben entspricht ungefähr der der Karpandji-Technik. In der Regel werden 2 Schrauben über dem Processus styloideus radii eingebracht, während eine 3. von dorsal aus nach palmar in das proximale Fragment platziert wird. Wir verwenden kanülierte Schrauben, die in minimalinvasiver Technik eingebracht 
werden. Eine rein perkutane Technik wird nicht empfohlen, da es durch die Drähte ebenso wie während des Eindrehens der Schrauben zu einer Verletzung von Sehnen kommen kann.

Indikation. Die Schraubenosteosynthese ist insbesondere bei A2-, B1-, B2- und C1Frakturen indiziert. Bei Osteoporose ist die Schraubenverankerung häufig nicht ausreichend, sodass sie nicht empfohlen werden kann. Bei jugendlichen, kooperativen Patienten kann auch die A3-Fraktur durch isolierte Schraubenosteosynthese sicher stabilisiert werden (• Abb. 1).

\section{Plattenosteosynthese}

Durch Einführen der winkelstabilen Osteosynthesesysteme hat sich die Konzeption bei der Behandlung von distalen Radiusfrakturen mit Hilfe der Osteosyntheseplatten deutlich gewandelt.

Dorsale Plattenosteosynthese. Bei nach dorsal dislozierten Frakturen wurde die Platte im Sinne einer Abstützosteosynthese folgerichtig dorsal platziert $[3,4]$. Die Komplikationsrate dieser Positionierung ist nicht unerheblich. Insbesondere werden Verletzungen der Sehnen beobachtet, v. a. die des M. extensor pollicis longus ist gefährdet. Eine entscheidende Verbesserung war durch Einführung der anatomisch geformten 2,4-mm-Plattensysteme möglich (z. B. Jupiterplatte). Die Ergebnisse mit diesem System sind sehr günstig. Allerdings ist der Zugang recht aufwändig und groß, zudem können auch bei diesem System Sehnenverklebungen vorkommen.

Volare Plattenosteosynthese. Volare Abstützplatten wurden in der Regel bei B3-Frakturen (Smith-Frakturen) routinemäßig angewandt. Seit Einführung der winkelstabilen 3,5-mm- und 2,4-mm-Platten kann die volare Plattenosteosynthese auch bei dorsal dislozierten Frakturen, insbesondere bei $\mathrm{A}_{3}$-Frakturen, eingesetzt werden $[2,3]$ (• Abb. 2). Mit dieser Technik werden sekundäre Dislokationen, wie sie für die konventionelle volare Plattenosteosynthese bei diesen Frakturen die Regel waren, nur sehr selten beobachtet. Wegen der guten muskulären Deckung

Trauma Berufskrankh 2006 · 8[Suppl 2]: S212-S217 DOI 10.1007/s10039-005-1061-3

(c) Springer Medizin Verlag 2006

\section{A. Dávid \\ Operative Therapie der distalen Radiusfraktur}

\section{Zusammenfassung}

Distale dislozierte Radiusfrakturen werden heute überwiegend operativ versorgt. Zunehmend werden die geschlossene Reposition und Kirschner-Draht-Osteosynthese durch eine interne Fixation mit Schrauben, winkelstabilen Platten oder einen Fixateur externe zurückgedrängt. Bei Frakturen mit schwerem Weichteilschaden wird das Handgelenk zunächst transfixiert, gleichzeit erfolgt die Rekonstruktion der Weichteile. Nach Weichteilkonsolidierung wird der Fixateur entweder durch eine interne Osteosynthese ersetzt oder durch Schrauben oder Kirschner-Drähte ergänzt, bis die Bruchheilung eingetreten ist. Es sollten möglichst nicht transfixierende

Montagen angewandt werden. Eine konservative Therapie kann heute nur noch bei stabilen oder nicht dislozierten Frakturen vertreten werden. Die arthroskopisch kontrollierte Rekonstruktion der distalen Radiusgelenkfläche wird heute eher kontrovers diskutiert und ist nicht als Standardverfahren akzeptiert.

\section{Schlüsselwörter}

Distale dislozierte Radiusfrakturen · Geschlossene Reposition · Kirschner-Draht-Osteosynthese · Interne Fixation · Interne Osteosynthese

\section{Surgical treatment of distal fracture of the radius}

\section{Abstract}

Distal dislocated radius fractures are now mostly treated surgically. Closed reduction and internal fixation with Kirschner wires are increasingly giving way to internal fixation with screws or fixed-angle plates and to the use of the fixateur externe. For fractures with concomitant severe soft tissue injury treatment with a bridging external fixator and adequate soft tissue management are first necessary. External fixation is needed in addition, however, once soft tissue repair has been achieved by means of internal fixation with screws or K-wires, and the external fixator should remain in place until the fracture has started to heal. If possible preference should be given to the use of nonbridging fixators. Conservative treatment can now no longer be justified except for stable and nondislocated fractures. Arthroscopy/assisted reconstruction of the carpal articular surface is the subject of some controversy and is not yet accepted as a standard procedure.

\section{Keywords}

Distal dislocated fractures of the radius . Closed reduction - Kirschner wires for internal fixation · Internal fixation 


\section{Körperferner Speichenbruch}

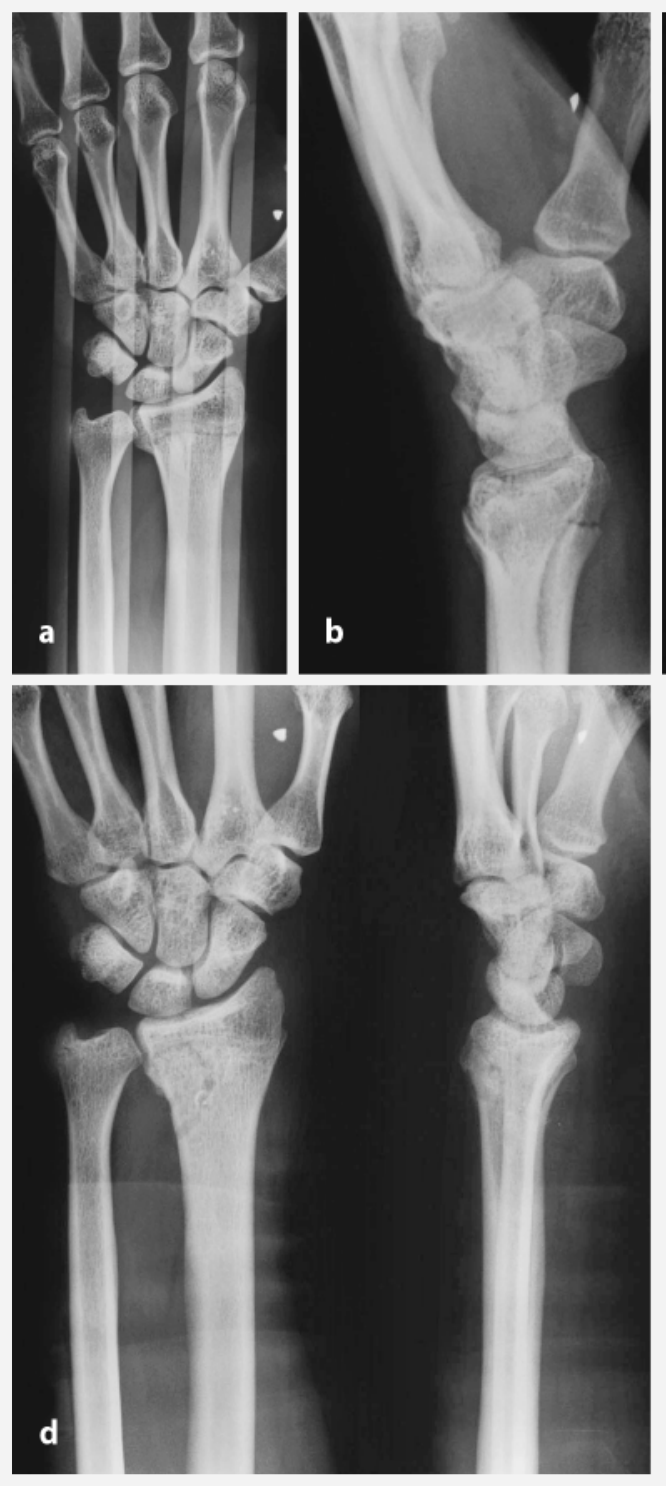

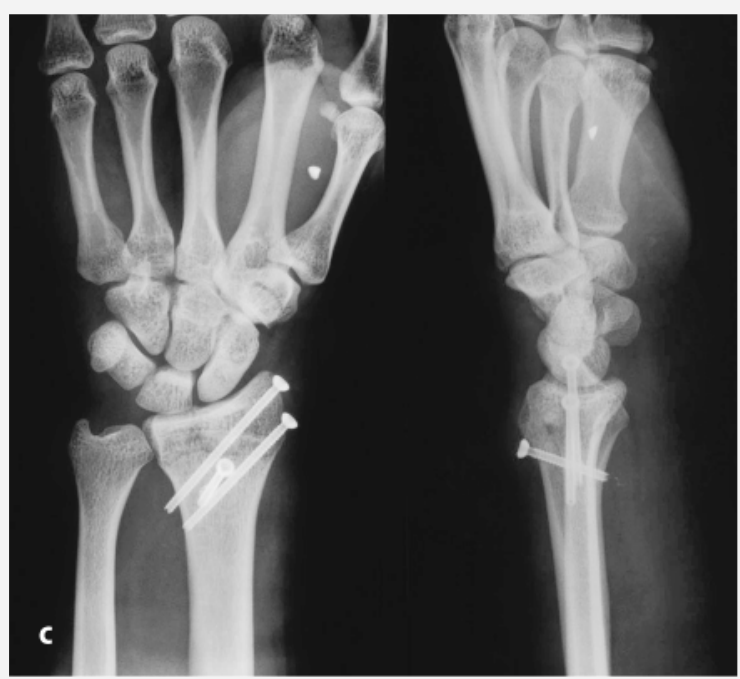

Abb. $1 \Delta$ Jugendlicher, A3Fraktur, a Unfallbild a.-p., b Unfallbild seitlich, c nach geschlossener Reposition und Schraubenosteosynthese, $\mathbf{d}$ Ausheilung nach ME der Platte sind Verklebungen mit den Flexorensehnen oder eine Schädigung des N. medianus extrem selten.

Eine besondere Schwierigkeit ist allerdings die Platzierung der distalen winkelstabilen Schrauben. Diese müssen subchondral liegen. Intraoperativ kann auch bei exakter Bildwandlerkontrolle nicht immer mit Sicherheit ausgeschlossen werden, dass die Gelenkfläche von den Schrauben tangiert oder perforiert wird. Bei C3-Frakturen oder sehr weit distal liegenden $\mathrm{A}_{3}$-Frakturen stößt diese Osteosynthesetechnik daher an ihre Grenzen.

Bei distalen Unterarmfrakturen ist es notwendig, auch die Ulna durch Plattenosteosynthese oder isolierte Schraubenosteosynthese oder eine 3,5-mm- oder
2,4-mm-Platte $\mathrm{zu}$ fixieren, wenn eine funktionelle Therapie angestrebt wird (• Abb.5). Ausnahme ist der isolierte Abriss des Griffelfortsatzes, der eine Abrissfraktur des ulnaren Seitenbands ist und keiner Refixation bedarf, wenn die Diastase $2-3 \mathrm{~mm}$ nicht überschreitet. Allerdings erfordert diese Fraktur ohne interne Fixation eine 4- bis 5-wöchige Ruhigstellung.

\section{Fixateur externe}

Transfixation des Handgelenks. Die Transfixation des Handgelenks über einen Fixateur externe mit Schanz-Schrauben im Radiusschaft und zweiten Mittelhandknochen ist die Standardtechnik zur Behandlung distaler Frakturen mit einem äußeren Fixationssystem [6]. Eine alleinige Transfixation führt aber nicht zu einer ausreichenden Stabilität im Bruchspalt, sodass stets eine additive Osteosynthese, entweder mit Bohrdrähten oder mit Schrauben, empfohlen wird (• Abb. 3). Die Indikation für diese Verfahren sind der schwere Weichteilschaden mit gleichzeitiger Spaltung der Unterarmkompartmente sowie der mehrfach verletzte Patient, bei dem eine provisorische Stabilisierung ohne großen Zeitverlust notwendig ist. Nach Stabilisierung des Patienten oder nach Weichteilkonsolidierung kann eine sekundäre interne Osteosynthese vorgenommen werden. 

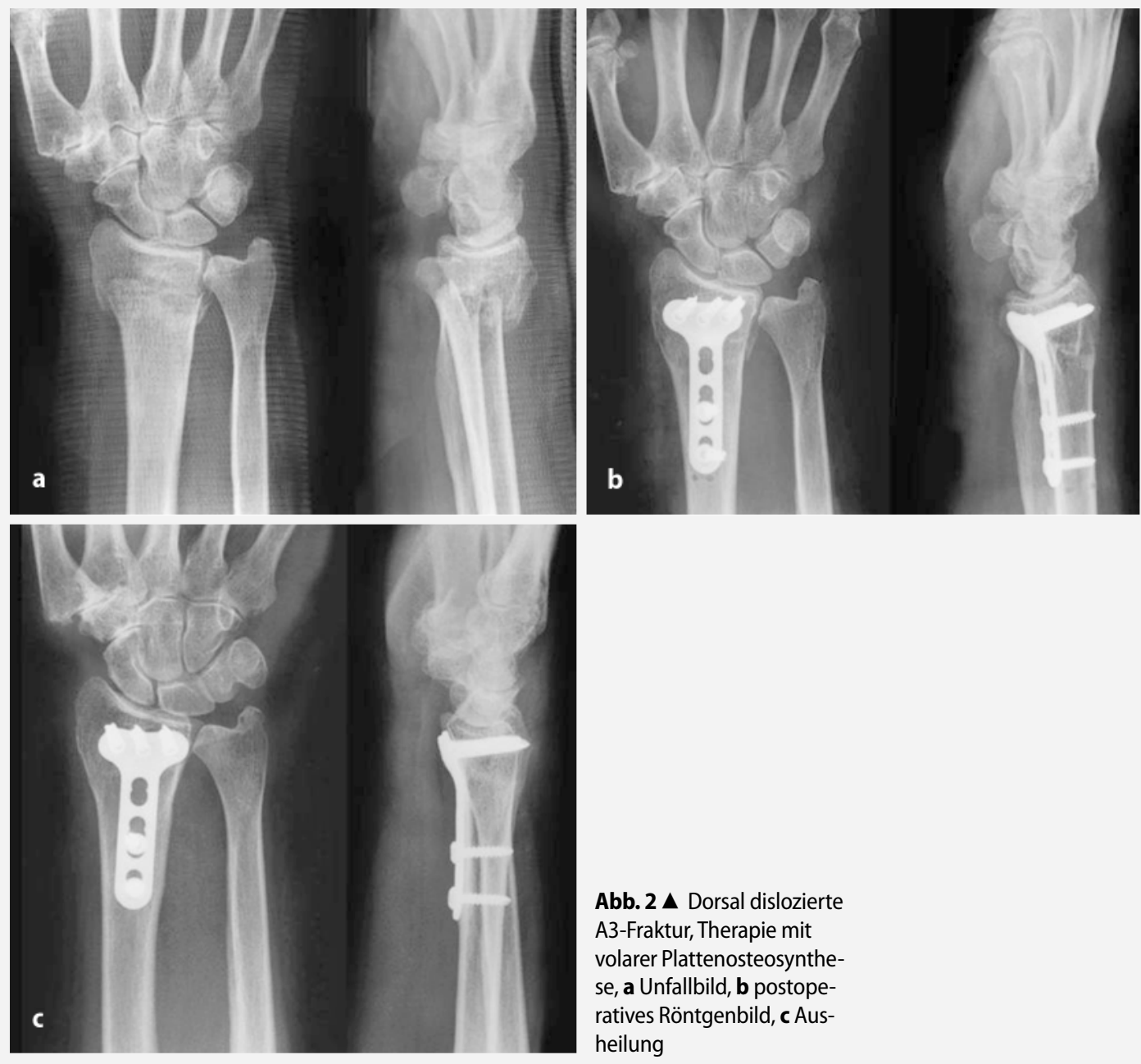

Abb. $2 \triangle$ Dorsal dislozierte

A3-Fraktur, Therapie mit

volarer Plattenosteosynthe-

se, a Unfallbild, b postope-

ratives Röntgenbild, c Aus-

heilung
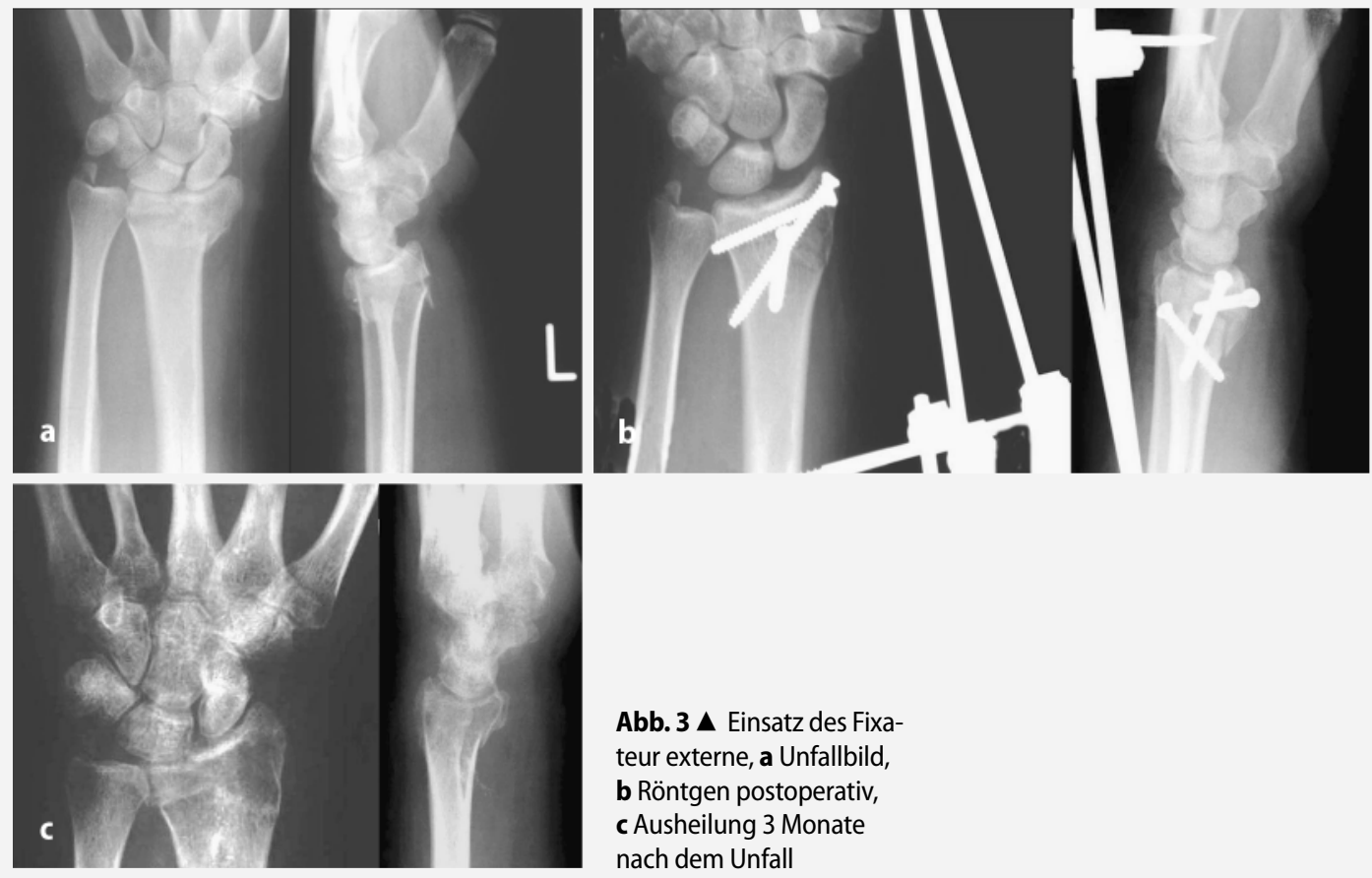

Abb. $3 \Delta$ Einsatz des Fixa-

teur externe, a Unfallbild,

b Röntgen postoperativ,

c Ausheilung 3 Monate

nach dem Unfall 


\section{Körperferner Speichenbruch}
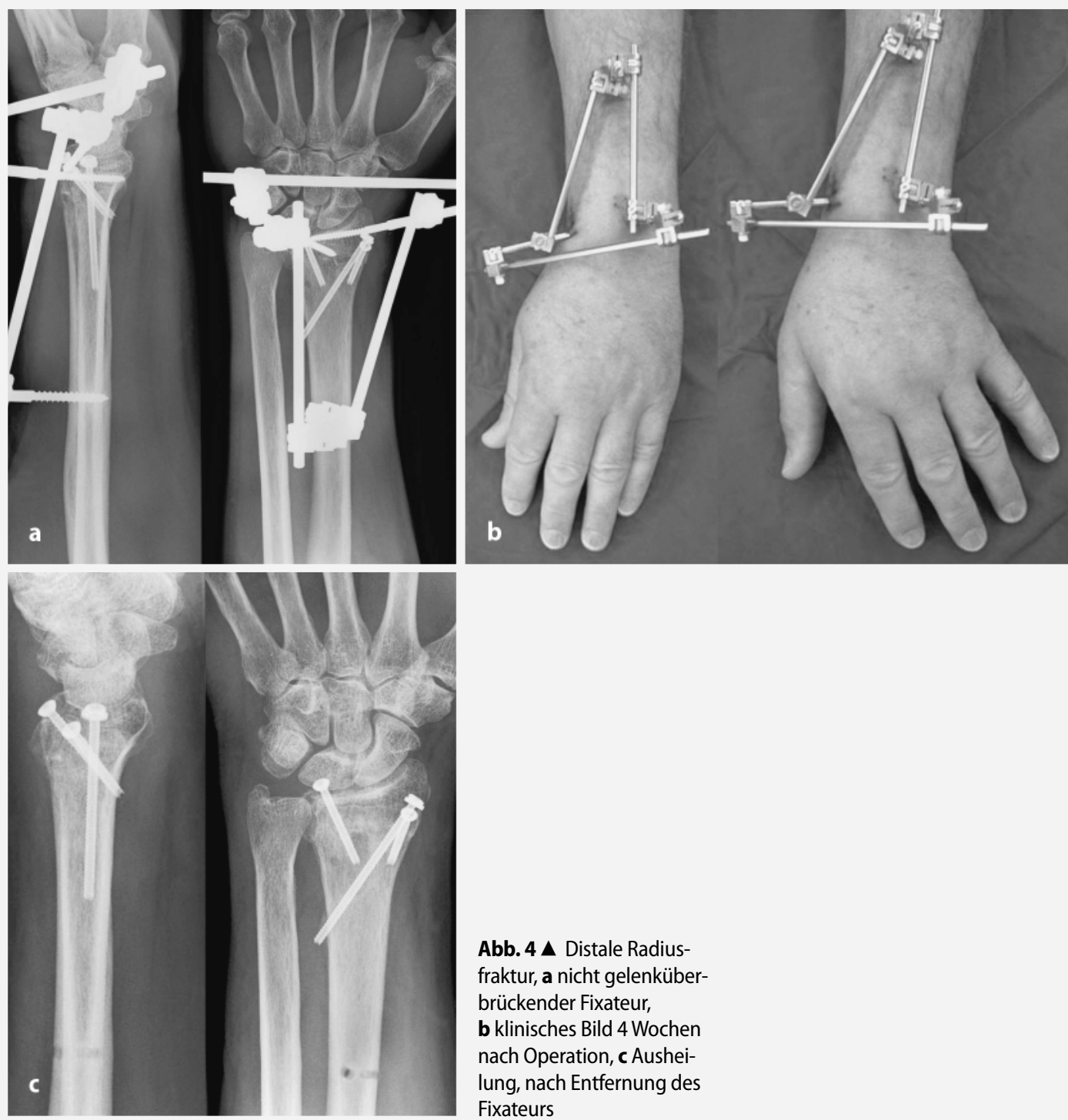

Abb. $4 \Delta$ Distale Radius-

fraktur, a nicht gelenküber-

brückender Fixateur,

b klinisches Bild 4 Wochen

nach Operation, c Aushei-

lung, nach Entfernung des

Fixateurs
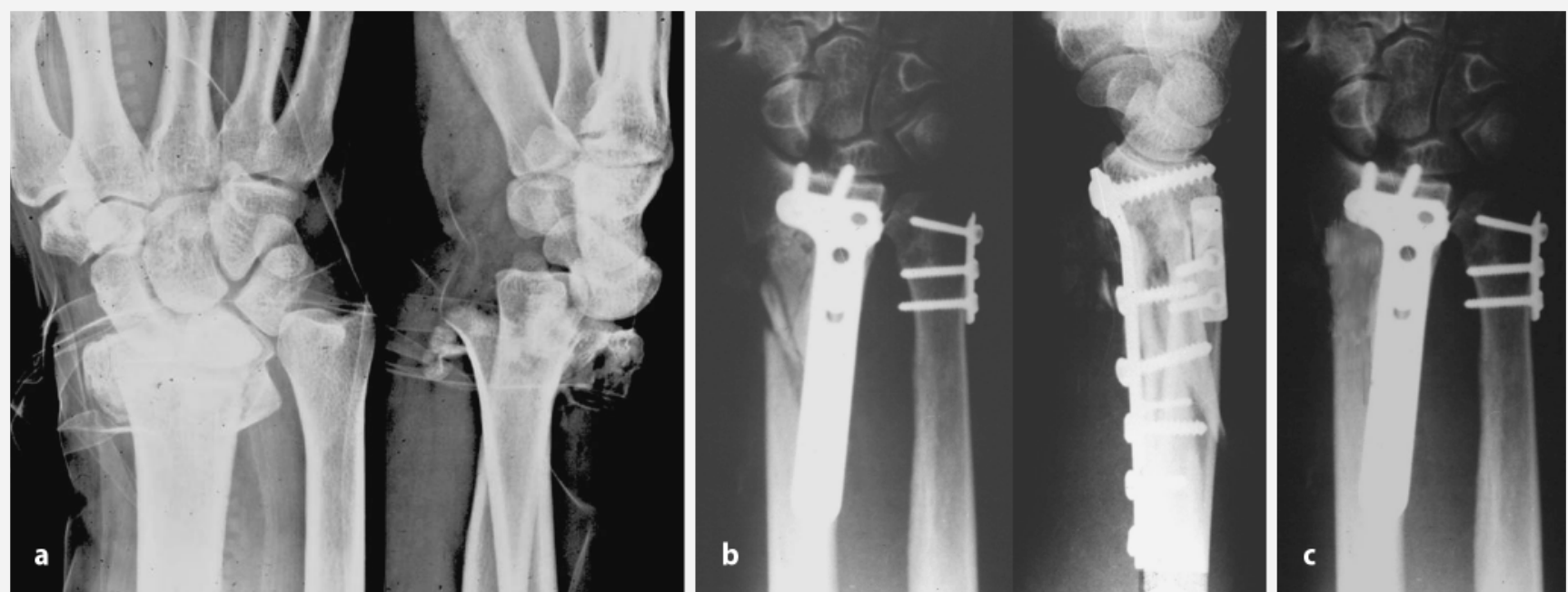

Abb. $5 \Delta$ Distale Unterarmfraktur, a Unfallbild, b Röntgen postoperativ, c Ausheilung 
Nicht gelenküberbrückender Fixateur externe. Neuere Entwicklungen ermöglichen es, die distale Radiusfraktur mit Schanz-Schrauben und gewindetragenden Kirschner-Drähten zu fixieren, ohne das Handgelenk zu transfixieren. Diese Verfahren werden derzeit weiterentwickelt, eine definitive Beurteilung, insbesondere im Vergleich zu den internen Osteosynthesen, steht noch aus (• Abb. 4). Da diese Technik überwiegend perkutan ausgeführt wird, sind Verletzungen der Sehnen im Bereich des Handgelenks möglich. Ein entscheidender Vorteil gegenüber der transfixierenden Technik ist, dass zumindest eine limitierte Bewegung des Handgelenks ermöglicht wird.

Bewegungsfixateure. Sie werden der komplexen Anatomie des Handgelenkes derzeit noch nicht vollständig gerecht. Sie ermöglichen einen eingeschränkten $\mathrm{Be}$ wegungsablauf für Dorsalextension und Palmarflexion.

Auch sie sollten mit einer internen Osteosynthese kombiniert werden, um im Bruchbereich eine ausreichende Stabilisierung der Fragmente zu erzielen.

\section{Wertung der einzelnen operativen Verfahren}

Sie ist außerordentlich schwierig. Es fehlt nicht nur an vergleichenden prospektiven Studien, sondern auch an langen Beobachtungszeiträumen. Ein direkter Vergleich der einzelnen Operationstechniken ist daher extrem erschwert. Zudem sind die Indikationen für die einzelnen Verfahren inkonstant. Das Ausmaß des Weichteilschadens und die Zertrümmerung, insbesondere der Gelenkfläche, sind in der Literatur z. T. nicht erwähnt oder nicht bewertet. Es besteht jedoch eindeutig ein Trend zu den winkelstabilen Plattenosteosynthesen, wobei zunehmend geringer dimensionierte Implantate verwendet werden. Die volare Position wird bevorzugt. Sehr gute Ergebnisse können aber auch nach dem derzeitigen Stand mit der anatomisch geformten dorsalen distalen Radiusplatte erzielt werden.

Die operative Versorgung dislozierter distaler Radiusfrakturen ( $\bullet$ Abb. 5) bestimmt heute den Goldstandard in der Unfallchirurgie. Eine konservative Thera- pie wird ausschließlich noch bei A2-Frakturen diskutiert.

Werden A3-Frakturen mit dorsal anatomisch geformten Platten, Schraubendurchmesser 2,4 $\mathrm{mm}$, oder mit volaren winkelstabilen Platten mit einem Schraubendurchmesser von 2,4 mm oder $3,5 \mathrm{~mm}$ versorgt, ist in der Regel eine funktionelle Nachbehandlung möglich. Bei Patienten mit festem Knochen kann alternativ auch die minimalinvasive Versorgung mit Schraubenosteosynthese erfolgen. Der Fixateur externe wird bei Frakturen mit schwerem Weichteilschaden zur temporären Transfixation des Handgelenks eingesetzt. In neuerer Zeit wurden Systeme entwickelt, die nicht gelenküberbrückend montiert werden können. Vorteil sind die minimalinvasive Implantation mit geringer Narbenbildung und der minimale Aufwand bei der Metallentfernung. Die isolierte, minimalinvasive Schraubenosteosynthese mit halboffener Reposition des distalen Radius hat sich bei den leichteren Frakturformen (A2-, B1-, B2-, C1-Frakturen) durchgesetzt. A3- und C2-Frakturen eignen sich nur bei festem Knochen für diese Technik.

Bei schwerem Weichteilschaden sollte die Fraktur zunächst mit einem Fixateur externe transfixiert werden, bis eine Weichteilkonsolidierung erreicht ist. Die alleinige Behandlung mit einem Fixateur externe reicht in der Regel nicht aus, um die Fraktur in achsgerechter Stellung zur Ausheilung zu bringen. Zumindest müssten additive interne Osteosynthesen durchgeführt oder aber grundsätzlich auf ein internes Stabilisierungssystem gewechselt werden.

Komplikationen der operativen Behandlungen. Sie müssen als eher gering angesehen werden. Neben den eher seltenen Infektionen $(<2 \%)$ sind es v. a. Sehnenverletzungen bei dorsalen Plattenosteosynthesen, die das funktionelle Ergebnis beeinträchtigen. Bei C-Frakturen muss auch mit einer hohen Inzidenz von posttraumatischen Arthrosen gerechnet werden. Die Algodystrophie ist ebenfalls eine nicht seltene Komplikation, die von der Bruchschädigung selbst sowie von Begleitverletzungen und dem Therapiemanagement induziert werden kann.

\section{Arthroskopie}

Die arthroskopisch kontrollierte Rekonstruktion der karpalen Gelenkfläche wird kontrovers diskutiert.

Als wesentliche Nachteile werden die ungünstige Übersicht auch bei Anwendung verschiedener Portale angeführt. Zudem ist die Technik eher schwierig und kann zu einer zusätzlichen Knorpel- und Bandschädigung führen. Derzeit kann auch nicht belegt werden, dass die Arthroskopie zu einer besseren Rekonstruktion der Gelenkfläche beiträgt [5].

\section{Metallentfernung}

Während volare Platten in der Regel nicht entfernt werden müssen, wird die Entfernung von isoliert liegenden Schrauben, insbesondere am Processus styloideus radii, empfohlen. Dies gilt auch für die dorsalen Platten, da hier immer mit einer Sehnenirritation durch das Implantat gerechnet werden muss.

Die anatomisch geformte dorsale Platte (Jupiterplatte) muss dagegen nach heutiger Erkenntnis nicht entfernt werden.

\section{Korrespondierender Autor}

\section{Prof. Dr. A. Dávid}

Zentrum für Unfallchirurgie und Orthopädische Chirurgie, Helios-Klinikum, Universitätsklinikum Witten/Herdecke Heusnerstraße 40, 42283 Wuppertal adavid@wuppertal.helios-kliniken.de

\section{Literatur}

1. Handholl HH, Madok R (2003) Conservative interventions for treating distal radial fractures in adults. Cochrane Database Syst Rev 2003: 2

2. Musgrave DS, Idler RS (2005) Volar fixation of dorsally displaced distal radius fractures using the 2.4$\mathrm{mm}$ locking compression plates. J Hand Surg [Am] 30: 743-749

3. Nana A, Joshi A, Lichtman DM (2005) Plating of the distal radius. J Am Acad Orthop Surg 13: 159-171

4. Ring D, McCarty LP, Campell D et al. (2004) Condylar blade plate fixation of unstable fractures of the distal ulna associated with fracture of the distal radius. J Hand Surg [Am] 29: 103-109

5. Ruch DS, Yang CC, Smith BP (2003) Results of acute arthroscopically repaired triangular fibrocartilage complex injuries associated with intra-articular distal radius fractures. Arthroscopy 19: 511-516

6. Ruch DS, Weiland AJ, Wolfe SW et al. (2004) Current concepts in the treatment of distal radial fractures. Instr Course Lect 53: 389-401

7. Siebert H (2005) Distale Radiusfrakturen. Unfallchirurg 108: 135-152 